\title{
Refeeding Syndrome in the Critically Ill: a Literature Review and Clinician's Guide
}

\author{
C. L. McKnight ${ }^{1} \cdot$ C. Newberry ${ }^{2} \cdot$ M. Sarav ${ }^{3} \cdot$ R. Martindale ${ }^{4} \cdot$ R. Hurt ${ }^{5} \cdot$ B. Daley ${ }^{6}$ \\ Published online: 22 November 2019 \\ (C) Springer Science+Business Media, LLC, part of Springer Nature 2019
}

\begin{abstract}
Purpose of Review To provide an overview of current methods of diagnosis and management of refeeding syndrome in the critically ill patient population.

Recent Findings Despite recent publications indicating refeeding syndrome (RFS) is an ongoing problem in critically ill patients, there is no standard for the diagnosis and management of this life-threatening condition. There is not a "gold standard" nutrition assessment tool for the critically ill. Currently, the National Institute for Health and Clinical Excellence criteria represent the best clinical assessment tool for RFS. Diagnosis and management with the help of a multidisciplinary metabolic team can decrease morbidity and mortality.

Summary Although a universal definition of RFS has yet to be defined, the diagnosis is made in patients with moderate to severe malnutrition who develop electrolyte imbalance after beginning nutritional support. The imbalances potentially can lead to cardiac, pulmonary, and gastrointestinal complications and failure. Standardizing a multidisciplinary nutrition care plan and formulating a protocol for critically ill patients who develop RFS can potentially decrease complication rates and overall mortality.
\end{abstract}

Keywords Refeeding syndrome $\cdot$ Malnutrition $\cdot$ Hypophosphatemia $\cdot$ Gastrointestinal $\cdot$ Enteral $\cdot$ Parenteral

\section{Introduction}

Appropriate nutritional support is crucial in the treatment of malnourished patients. Regardless of the disease associated

This article is part of the Topical Collection on Nutrition and Obesity

C. L. McKnight

cmcknight@utmck.edu

1 Department of Trauma/Surgical Critical Care, University of Tennessee-Knoxville, 1924 Alcoa Highway Box U-11, Knoxville, TN 37920, USA

2 Division of Gastroenterology \& Hepatology, Weill Cornell Medical Center, 1283 York Avenue, 9th Floor, New York, NY 10065, USA

3 Section of Nephrology and Hypertension, NorthShore University Health Systems, University of Chicago, Chicago, USA

4 Department of General Surgery, Oregon Health Science University, 3303 SW Bond Ave Fl 6, Portland, OR 97239, USA

5 Division of Gastroenterology \& Hepatology, Mayo Clinic, Rochester, MN, USA

6 Department of Trauma/Surgical Critical Care, University of Tennessee Graduate School of Medicine, 1924 Alcoa Highway Box U-11, Knoxville, TN 37920, USA with the patient's moderate or severe protein-calorie malnutrition, clinicians can anticipate a significant delay in the patient's clinical response to sufficient protein and calories. There are potential deleterious effects of feeding the malnourished patient, the most common being refeeding syndrome.

\section{Definition of Refeeding Syndrome}

Although the constellation of symptoms was identified in the Napoleonic era of the 19th century, refeeding syndrome (RFS) was not reported until the 1940s when malnourished prisoners from World War II developed cardiac and neurological disorders after resuming nutrition. As it is not easy to isolate exactly when refeeding syndrome was first discovered, it is just as difficult to define RFS even among nutrition experts. "There is not an international consensus on the definition of refeeding syndrome [1]." Before clearly defining RFS, the mechanisms that occur with refeeding need to be understood.

Friedli et al. published the first systematic review in March 2017 with the objective to define RFS and develop a method of treatment including prevention. The definition of RFS varied in the literature and focused on electrolyte 
disturbances [2•]. Most clinicians associate RFS with several clinical features, such as severe electrolyte and fluid shifts making it a potentially lethal condition. RFS is associated with severe metabolic abnormalities, such as glucose metabolism imbalance, hypophosphatemia, hypokalemia, hypomagnesemia, and thiamine deficiency regardless of whether the nutrition is provided enterally or parenterally [3].

\section{Incidence}

Because there is no definition, the incidence of RFS is unknown [4•]. Much of the published literature does not report the overall incidence of RFS, but several articles narrow it down to the incidence of hypophosphatemia. Malnourished patients have a 60 times greater chance of developing hypophosphatemia. Severe hypophosphatemia has an allcause mortality of $18.2 \%$ compared with $4.6 \%$ among patients without hypophosphatemia. Critically ill patients who have had nutrition held for $48 \mathrm{~h}$ have a $34 \%$ chance of becoming hypophosphatemic [5]. The systematic reviews of Friedli et al., Rio et al., and Camp et al. report an overall incidence rate of $0-2 \%$, but other studies have reported anywhere from 50 to $80 \%[2 \bullet, 6,7]$. This drastic discrepancy in incidence is due to the unclear definition to diagnose RFS.

Specific subset populations within the ICU are at risk for RFS. The incidence of RFS in cancer patients can be as high as $25 \%$ [8]. It is present in patients with eating disorders, short bowel syndrome, chronic alcohol and drug use, failure to thrive, kwashiorkor/marasmus, chronic diabetics, chronic diuretic use, inflammatory bowel disease, chronic pancreatitis, long-term antacid use, hyperemesis gravidarum, chronic infectious disease such as AIDS, tuberculosis, cystic fibrosis, or congenital heart disease. RFS is also common in postoperative, elderly, morbidly obese with profound weight loss, the critical ill, and the homeless patient population [4•].

\section{Risk Factors for RFS}

Any patient who has had little or no nutritional intake for several consecutive days or is metabolically stressed from critical illness or a major surgery is at risk of refeeding syndrome. Chen et al. addresses risk factors, treatment, and prevention of RFS by referencing the NICE clinical nutrition guidelines in the "high-risk" chronic critically ill population. The treatment regimen included low calorie nutritional support initially with slow increases to total estimated requirement after resuscitation in Table 1 [9].

The NICE criteria were derived from the guidelines of the National Institute for Health and Clinical Excellence to identity patients at high risk of refeeding problems. Unfortunately, recommendations are from low-grade evidence, mainly cohort and case series studies, but they are based on the best evidence that is available. Patients at risk for RFS include one or more of the following criteria in Table 2 : BMI $<16 \mathrm{~kg} / \mathrm{m}^{2}$, prolongation of insufficient nutrition for more than 10 days, more than $15 \%$ weight loss in 3-6 months, and decreased serum electrolyte-vitamin levels before nutrition is started or two or more of the following criteria: BMI $<18.5 \mathrm{~kg} / \mathrm{m}^{2}$, unintentional weight loss of $>10 \%$ in $3-6$ months, minimal or no nutritional intake for $>5$ days, and history of alcohol or drug use $[4 \bullet, 8,10,11 \bullet \bullet]$.

Marik et al. performed one of the few prospective ICU studies that attempted to identify risk factors for RFS. The only predictive risk factor for refeeding-related hypophosphatemia was a serum prealbumin $<110 \mathrm{~g} / \mathrm{L}$. [5] Low serum magnesium $<0.7 \mathrm{mmol} / \mathrm{L}$ was found to be a predictor in Rio et al. [7, 10]. The NRS-2002 and the Nutric Score are screening tools developed from analysis of controlled clinical trials to stratify risk of malnutrition. These currently are our best inpatient assessment tools to delineate malnourished patients in the intensive care unit [12•].

\section{Pathophysiology}

During a fasting period, the body adapts from carbohydrates to fatty and amino acids for the main energy source. The basal metabolic rate (BMR) drastically decreases [12•, 13]. Many intracellular minerals become severely depleted during this period without exogenous supply. Most importantly, insulin secretion is suppressed in this fasted state and glucagon secretion is increased $[4 \bullet, 13]$. The release of glucagon drives metabolism.

The reintroduction of nutrition to a starved or fasted individual results in a rapid decline in both gluconeogenesis and anaerobic metabolism. During refeeding, there is a shift back from free fatty acids and ketone bodies to carbohydrate metabolism along with insulin secretion increasing in response to increased blood sugar; thus, glycogen, fat, and protein synthesis are increased. This process requires phosphate, magnesium, and potassium, which were depleted and the remaining stores are rapidly utilized, leading to low extracellular levels of magnesium, phosphorus, and calcium by active and passive transport into the intracellular compartment. Since the intracellular compartment was depleted and a wide concentration gradient exists, a rapid depletion of the extracellular ions occurs. To maintain the neutral osmotic gradient, positive ion homeostasis, sodium is retained and hence water follows causing hypervolemia and edema resulting in anasarca. Hypervolemia and shifting of electrolytes increase cardiac workload and heart rate. This leads to acute heart failure. Oxygen consumption increases which stresses multiple systems and can prolong respiratory failure. 
Table 1 Total estimated requirement after resuscitation

\begin{tabular}{|c|c|c|c|c|}
\hline & & Mild risk & Moderate risk & Severe risk \\
\hline \multirow[t]{5}{*}{ Day 1} & Nutrition & $15-25 \mathrm{kcal} / \mathrm{kg} / \mathrm{day}$ & $10-15 \mathrm{kcal} / \mathrm{kg} / \mathrm{day}$ & $5-10 \mathrm{kcal} / \mathrm{kg} /$ day \\
\hline & Fluid management & $30-35 \mathrm{~mL} / \mathrm{kg} /$ day & $25-30 \mathrm{~mL} / \mathrm{kg} /$ day & $20-25 \mathrm{~mL} / \mathrm{kg} /$ day \\
\hline & Sodium & No restriction & $<1 \mathrm{mmol} / \mathrm{kg} /$ day & $<1 \mathrm{mmol} / \mathrm{kg} /$ day \\
\hline & Thiamine $200-300$ & Yes & Yes & Yes \\
\hline & MVI & Yes & Yes & Yes \\
\hline \multirow[t]{5}{*}{ Day 2} & Nutrition & $15-25 \mathrm{kcal} / \mathrm{kg} /$ day & $10-15 \mathrm{kca} / \mathrm{kg} / \mathrm{day}$ & 5-10 kcal/kg/day \\
\hline & Fluid management & $30-35 \mathrm{~mL} / \mathrm{kg} /$ day & $25-30 \mathrm{~mL} / \mathrm{kg} /$ day & $20-25 \mathrm{~mL} / \mathrm{kg} /$ day \\
\hline & Sodium & No restriction & $<1 \mathrm{mmol} / \mathrm{kg} /$ day & $<1 \mathrm{mmol} / \mathrm{kg} /$ day \\
\hline & Thiamine $200-300$ & $200-300 \mathrm{mg}$ & Yes & Yes \\
\hline & MVI & Yes & Yes & Yes \\
\hline \multirow{5}{*}{ Day 3} & Nutrition & $15-25 \mathrm{kcal} / \mathrm{kg} /$ day & $10-15 \mathrm{kca} / \mathrm{kg} / \mathrm{day}$ & $5-10 \mathrm{kcal} / \mathrm{kg} /$ day \\
\hline & Fluid management & $30-35 \mathrm{~mL} / \mathrm{kg} /$ day & $25-30 \mathrm{~mL} / \mathrm{kg} /$ day & $20-25 \mathrm{~mL} / \mathrm{kg} /$ day \\
\hline & Sodium & No restriction & $<1 \mathrm{mmol} / \mathrm{kg} /$ day & $<1 \mathrm{mmol} / \mathrm{kg} /$ day \\
\hline & Thiamine $200-300$ & $200-300$ & Yes & Yes \\
\hline & MVI & Yes & Yes & Yes \\
\hline \multirow[t]{5}{*}{ Day 4} & Nutrition & $30 \mathrm{kcal} / \mathrm{kg} /$ day & $15-25 \mathrm{kcal} / \mathrm{kg} / \mathrm{day}$ & $10-20 \mathrm{kcal} / \mathrm{kg} /$ day \\
\hline & Fluid management & $30-35 \mathrm{~mL} / \mathrm{kg} /$ day & $30-35 \mathrm{~mL} / \mathrm{kg} /$ day & $25-30 \mathrm{~mL} / \mathrm{kg} /$ day \\
\hline & Sodium & No restriction & $<1 \mathrm{mmol} / \mathrm{kg} /$ day & $<1 \mathrm{mmol} / \mathrm{kg} /$ day \\
\hline & Thiamine & No & No & Yes \\
\hline & MVI & Yes & Yes & Yes \\
\hline \multirow[t]{5}{*}{ Day 5} & Nutrition & Full requirements & $15-25 \mathrm{kcal} / \mathrm{kg} / \mathrm{day}$ & $10-20 \mathrm{kcal} / \mathrm{kg} /$ day \\
\hline & Fluid management & $30-35 \mathrm{ml} / \mathrm{kg} /$ day & $30-35 \mathrm{~mL} / \mathrm{kg} /$ day & $25-30 \mathrm{~mL} / \mathrm{kg} /$ day \\
\hline & Sodium & No restriction & $<1 \mathrm{mmol} / \mathrm{kg} /$ day & $<1 \mathrm{mmol} / \mathrm{kg} /$ day \\
\hline & Thiamine & No & No & Yes \\
\hline & MVI & Yes & Yes & Yes \\
\hline \multirow[t]{5}{*}{ Day 6} & Nutrition & Full requirements & $25-30 \mathrm{kcal} / \mathrm{kg} / \mathrm{day}$ & $10-20 \mathrm{kcal} / \mathrm{kg} /$ day \\
\hline & Fluid management & $30-35 \mathrm{ml} / \mathrm{kg} / \mathrm{d}$ & $30-35 \mathrm{~mL} / \mathrm{kg} /$ day & $25-30 \mathrm{~mL} / \mathrm{kg} /$ day \\
\hline & Sodium & No restriction & $<1 \mathrm{mmol} / \mathrm{kg} /$ day & $<1 \mathrm{mmol} / \mathrm{kg} /$ day \\
\hline & Thiamine & No & No & No \\
\hline & MVI & Yes & Yes & Yes \\
\hline \multirow[t]{5}{*}{ Day 7} & Nutrition & Full requirements & Full requirements & $20-30 \mathrm{kcal} / \mathrm{kg} /$ day \\
\hline & Fluid management & $30-35 \mathrm{ml} / \mathrm{kg} /$ day & $30-35 \mathrm{~mL} / \mathrm{kg} /$ day & $30-35 \mathrm{~mL} / \mathrm{kg} /$ day \\
\hline & Sodium & No restriction & $<1 \mathrm{mmol} / \mathrm{kg} /$ day & $<1 \mathrm{mmol} / \mathrm{kg} /$ day \\
\hline & Thiamine & No & No & No \\
\hline & MVI & Yes & Yes & Yes \\
\hline \multirow[t]{5}{*}{ Day 8} & Nutrition & Full requirements & Full requirements & $20-30 \mathrm{kcal} / \mathrm{kg} /$ day \\
\hline & Fluid management & $30-35 \mathrm{ml} / \mathrm{kg} /$ day & $30-35 \mathrm{~mL} / \mathrm{kg} /$ day & $30-35 \mathrm{~mL} / \mathrm{kg} /$ day \\
\hline & Sodium & No restriction & No restriction & $<1 \mathrm{mmol} / \mathrm{kg} /$ day \\
\hline & Thiamine & No & No & No \\
\hline & MVI & Yes & Yes & Yes \\
\hline \multirow[t]{5}{*}{ Day 9} & Nutrition & Full requirements & Full requirements & $20-30 \mathrm{kcal} / \mathrm{kg} /$ day \\
\hline & Fluid management & $30-35 \mathrm{ml} / \mathrm{kg} /$ day & $<1 \mathrm{mmol} / \mathrm{kg} /$ day & $30-35 \mathrm{~mL} / \mathrm{kg} /$ day \\
\hline & Sodium & No restriction & No restriction & $<1 \mathrm{mmol} / \mathrm{kg} /$ day \\
\hline & Thiamine & No & No & No \\
\hline & MVI & Yes & Yes & Yes \\
\hline \multirow[t]{5}{*}{ Day 10} & Nutrition & Full requirements & Full requirements & Full requirements \\
\hline & Fluid management & $30-35 \mathrm{ml} / \mathrm{kg} /$ day & $<1 \mathrm{mmol} / \mathrm{kg} /$ day & $30-35 \mathrm{~mL} / \mathrm{kg} /$ day \\
\hline & Sodium & No restriction & No restriction & $<1 \mathrm{mmol} / \mathrm{kg} /$ day \\
\hline & Thiamine & No & No & No \\
\hline & MVI & Yes & Yes & Yes \\
\hline
\end{tabular}

$\mathrm{BMI} \leq 14$ should be on cardiac monitoring

Clinician should make modifications based on clinical presentation such as edema, tachycardia, and tachypnea

Formation of phosphorylated carbohydrate compounds in the liver and skeletal muscle depletes intracellular ATP and 2,3-diphosphoglycerate (DPG) in red blood cells, leading to cellular dysfunction and inadequate oxygen delivery to the body's organs. Refeeding overall increases a patient's BMR. During the refeeding process, patients become energy inefficient due to hypermetabolism and increase dietary-induced thermogenesis. Patients being refed require more energy per kilogram of body weight than calculated in standard formulae.

Cardiac arrhythmias are the most common cause of death from refeeding syndrome; other risks include confusion, coma, seizures, and respiratory and cardiac failure. Reactivation of carbohydrate-dependent metabolic pathways increases the demand for thiamine, which acts as a cofactor for certain enzyme; 
Table 2 Inclusion criteria for RFS

\begin{tabular}{lcc}
\hline Minor (A) & Major (B) & Very high risk $(\mathrm{C})$ \\
\hline BMI $<18.5 \mathrm{~kg} / \mathrm{m}^{2}$ & $\mathrm{BMI}<16 \mathrm{~kg} / \mathrm{m}^{2}$ & $\mathrm{BMI}<14 \mathrm{~kg} / \mathrm{m}^{2}$ \\
$>10 \%$ unintentional & $>15 \%$ unintentional & $>20 \%$ unintentional \\
weight loss within & weight loss within & weight loss within \\
3-6 months & $3-6$ months & $3-6$ months \\
Minimal nutritional & Minimal nutritional & Minimal nutritional \\
intake $>5$ days & intake $>10$ days & intake $>15$ days \\
History of alcohol or & $\begin{array}{l}\text { Initial low levels of } \\
\text { drug abuse }\end{array}$ & potassium, \\
& phosphate, or & \\
& magnesium &
\end{tabular}

National Institute for Clinical Excellence (NICE) guidelines for management of refeeding syndrome: use for identifying patients at risk for refeeding syndrome $[2 \cdot]$

therefore, $\mathrm{B}_{1}$ vitamin thiamine may fall. Thiamine is an essential water absorbable nutrient that has a short half-life between 9 and 18 days. It is a key component of aerobic cellular respiration, so deficiency can be devastating.

Thiamine diphosphate (TDP) and thiamine pyrophosphate (TPP) are thiamine derivatives that serve as coenzymes in four enzymatic reactions including pyruvate dehydrogenase complex, $\alpha$-ketoglutarate dehydrogenase, $\alpha$-keto-acid dehydrogenase complex, and glutamic acid and gamma-aminobutyric acid (GABA) inhibitory neurotransmittors. Pyruvate dehydrogenase facilitates glycolysis to the TCA cycle, producing Acetyl-CoA. Another enzymatic reaction that involves thiamine is $\alpha$-Ketoglutarate dehydrogenase complex, which is the rate-limiting step of the TCA cycle. The pentose phosphate shunt requires thiamine for transketolase involved in nucleic acid and lipid production. Thiamine is also required for the branched-chain $\alpha$-keto-acid dehydrogenase complex for the metabolism of branched-chain amino acids. Thiamine is involved in production of neurotransmitters such as glutamic acid, which is an excitatory neurotransmitter for the central nervous system. At the synapses, glutamate is released in vesicles from the presynaptic cell and bind G-protein-coupled receptors on the postsynaptic cells, known as N-methyl-Daspartate (NMDA) receptors. The activity of glutamic acid on the ion channel receptors promotes cognitive functions, such as learning and memory. Glutamate is also a precursor for the synthesis of GABA, an inhibitory neurotransmitter.

The deficiency of thiamine leads to beriberi, which presents as wet or dry. Dry beriberi usually manifests within the nervous system, often times as peripheral neuropathy. WE is another presentation of dry beriberi. Wernicke's encephalopathy (WE) is characterized by the triad of ocular abnormalities, ataxia, and a global confused state [14]. Frequently associated with chronic alcohol abuse, WE occurs in malnourished patients who receive carbohydrate loading with minimal thiamine storage. The brain is affected because TPP is required for carbohydrate metabolism and the brains primary energy source is glucose. A combination of oxidative stress, excitotoxicity, inflammation, decline in neurogenesis, disruption of blood-brain barrier, lactic acidosis, diminished astrocyte functional integrity, and loss of glutamate transporters occurs with thiamine deficiency $[15,16]$. Providing adequate thiamine before starting nutrition can prevent WE.

If cardiovascular compromise is associated with thiamine deficiency, then the term is wet beriberi. The chronic form presents with peripheral vasodilation, decreased vascular resistance, and subsequent fluid retention through the reninangiotensin-aldosterone system. These patients have volume overload and peripheral edema. The acute form presents with tachycardia, hypotension, and lactic acidosis and may not be characterized with peripheral edema, but demonstrates left ventricular heart dysfunction [15].

The depletion of phosphate, magnesium, potassium, and thiamine occurs at varying degrees and therefore the clinical effects vary. Chronic alcohol abusers or those with long-term starvation are more vulnerable to the metabolic consequences of multiple independent mineral and elemental deficiencies. This explains why RFS does not have a set definition. The presentation of signs and symptoms varies secondary to a broad spectrum of biochemical abnormalities. If the electrolyte imbalances are mild, then the clinical picture may be mild. The clinical presentation can be anywhere along the spectrum from nausea and vomiting to possibly even death. Most electrolyte disturbances occur within the first 2-3 days of refeeding but can occur up to $7-10$ days later.

\section{Enteral vs Parenteral}

Enteral nutrition (EN) is preferred over parenteral nutrition (PN) for the benefits on intestinal and immunologic function. Early EN reduces infectious complications in moderate to severely injured trauma patients compared with PN or starvation for 5 days. Enteral nutrition is preferable to parenteral nutrition in critically ill patients due to this reduction in infectious complications, a decreased stress response with EN compared to PN, maintenance of enterocyte viability and growth, and the promotion of a strong gut mucosal barrier.

Although the 2016 ASPEN/SCCM guidelines suggest that RFS is more common with PN than EN, evidence by Zekei et al. suggests just the opposite. Evidence suggests it is more common with EN. The likely mechanism is explained by a greater increase in GLP-1 or Incretin levels in response to EN. This raises insulin to higher levels than that seen with PN. Permissive underfeeding and reaching caloric goals slowly over 3-4 days by a protocol can decrease complications [11••, 17].

The 2016 ASPEN guidelines recommend hypocaloric PN dosing of $\leq 20 \mathrm{kcal} / \mathrm{kg}$ day or $80 \%$ of estimated energy needs in severely malnourished patients that have contraindications 
Fig. 1 The 2016 ASPEN guidelines recommend NRS2002

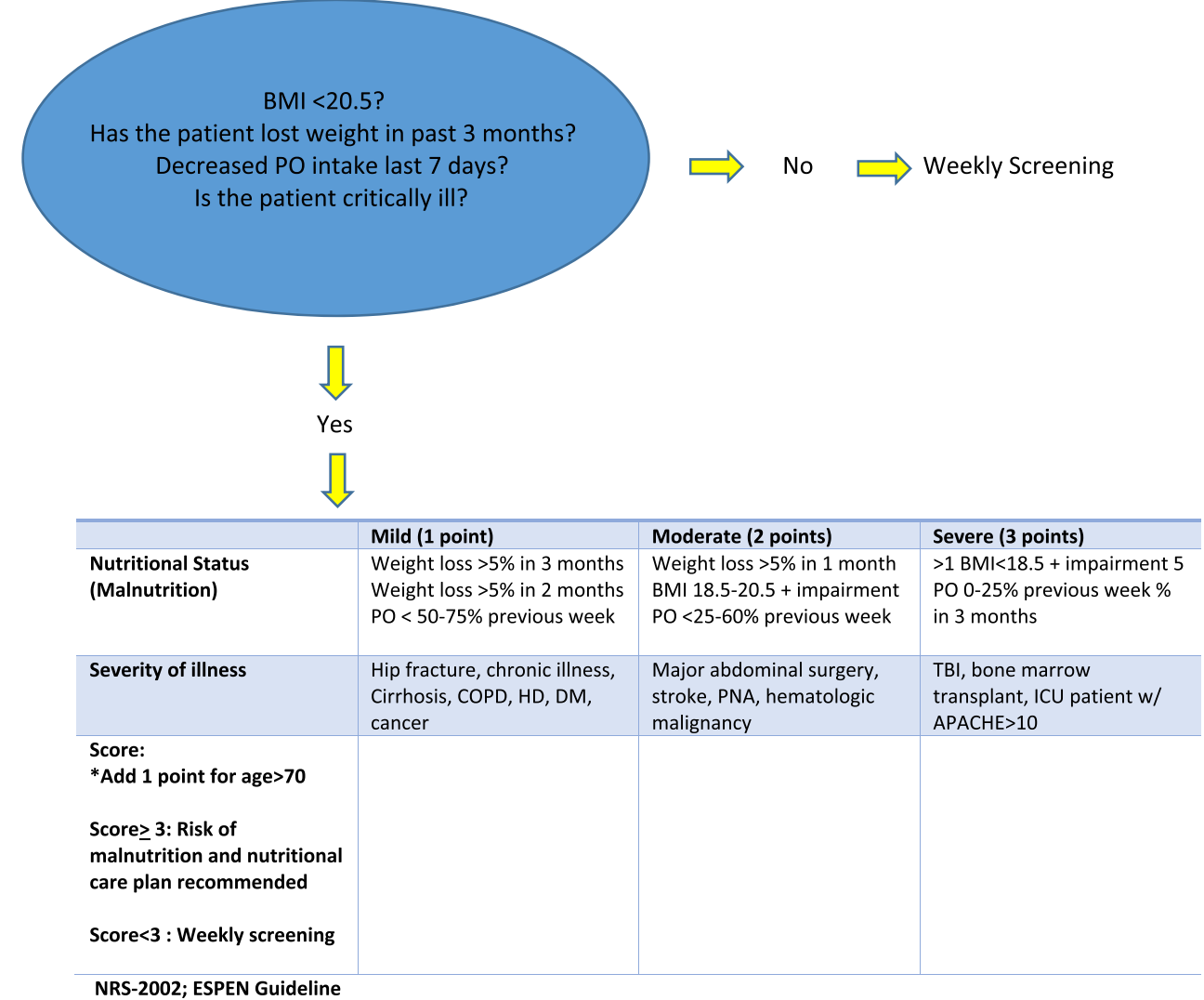

to EN over the first week of hospitalization in the ICU. Protein requirements should not be reduced. This not only decreases complications associated with RFS, but also reduces hyperglycemia and insulin resistance, infection rates, ventilator days, and hospital stay $[11 \bullet \cdot]$.

\section{Outcomes}

Very few studies address the overall outcome of RFS. Doig et al. performed a randomized, multicenter, single-blinded controlled trial involving 13 hospital ICUs in Australia and New Zealand looking at restricted versus standard nutritional support in critically ill patients who developed RFS $(\leq 0.32$ $\mathrm{mmol} / \mathrm{l}$ phos) within $72 \mathrm{~h}$ of starting nutrition. Even though caloric restriction (20 kcal/h for 2 days) meant a longer hospital length of stay, the overall 60- and 90-day survival rate improved when compared with the standard group. When the phosphorus levels were improving, the calories were gradually increased over the next 4 days. If the phosphorus was less than $0.71 \mathrm{mmol} / 1$ then the calories were reduced to $20 \mathrm{kcal} / \mathrm{h}$. Even though the trial did not show a difference in the mean number of days alive 60 days after ICU discharge, it did show more patients were alive at day 60 after ICU discharge and the overall survival time was increased in the caloric restricted group $[18 \bullet \bullet]$.
Olthof et al. showed there was no difference in 3 or 6 month mortality rates, ICU length of stay, or ventilator days between patients with RFS and patients without RFS, but when comparing the results of permissive hypocaloric feeding between the RFS and the non-RFS groups, the former had significant decrease in overall mortality rates at 6 months [19].

These two studies form the mainstay of the ICU refeeding guidelines. The results are impossible to compare because each study used completely different study designs and used different definitions of RFS and serum phosphorus level cut offs $[18 \bullet \bullet, 19 \bullet, 20 \bullet$. We, however, draw the conclusion that hypocaloric feeding improves outcomes in RFS.

\section{Management and Treatment}

Multidisciplinary metabolic support teams positively impact overall patient outcomes [21, 22]. The National Institute for Health and Clinical Excellence guidelines recommend all critically ill patients should have baseline laboratory values checked and undergo a risk assessment tool that factors in the severity of illness $[2 \bullet, 4 \bullet, 23 \bullet \bullet]$. The 2016 ASPEN guidelines recommend NRS-2002 (Fig. 1) or NUTRIC score to assess for severity of malnourishment and the National Institute for Health and Clinical Excellence recommends the NICE criteria to assess risk of developing refeeding 
syndrome. Once mild, moderate, or severe risk is established, the rate of nutrition, fluid, number of days of sodium restriction, thiamine, and multivitamin administration can be determined (Table 1) $[11 \bullet \cdot, 12 \bullet, 23 \bullet \cdot]$.

Thiamine must be administered at least $30 \mathrm{~min}$ before nutrition is initiated to prevent Wernicke's encephalopathy [17]. ECG monitoring is recommended in patients with severe risk for RFS. If $\mathrm{K}<2.5 \mathrm{mmol} / \mathrm{L}, \mathrm{PO}_{4}<0.32 \mathrm{mmol} / \mathrm{L}, \mathrm{Mg}<0.5$ $\mathrm{mmol} / \mathrm{L}$, a cardiac monitor should be considered $[2 \bullet, 17]$. A multivitamin should be administered daily for at least 10 days. Sodium intake should be restricted if edema occurs. Fluid intake should be closely monitored. Rapid correction of electrolyte disturbances while feeding is best practice. NICE indicates that feeding and correction of biochemical abnormalities can occur in tandem without deleterious effects to the patient $[2 \bullet, 10,11 \bullet, 14,24]$.

If discovered to be a higher risk for RFS, nutritional depletion should be deliberate with a maximum rate of 10 $\mathrm{kcal} / \mathrm{kg}$ every $24 \mathrm{~h}$. The goal rate can be met within 4 to 7 days. If the BMI is $\leq 14 \mathrm{~kg} / \mathrm{m}^{2}$ or no nutrition for 14 days, then feeding should be started at $5 \mathrm{kcal} / \mathrm{kg} / 24 \mathrm{~h}$. Electrolyte derangements will occur in RFS and should be checked daily for the first week, and at least three times the second week [4•]. PN should be slowly advanced for patients in the ICU at risk for RFS. Electrolytes such as phosphate and magnesium should be checked every $12 \mathrm{~h}$ after PN has started for the first 3 days [25].

\section{Conclusions}

The definition of RFS varies and is ostensibly a clinical definition based on global symptoms of electrolyte abnormalities in previously malnourished patients. RFS has been recognized for centuries but adequate treatments were only first recognized around World War II. Even though there is not a "gold standard" assessment tool, NRS-2002 or Nutric Score assess severity of malnutrition with severity of disease as a factor. NICE criteria can identify and stratify who may be at risk for refeeding syndrome. Close monitoring and development of a thoughtful plan with hypocaloric feeding will likely prevent the extremes of RFS in highrisk patients. Multidisciplinary metabolic support teams are recommended to optimize patient outcomes.

\section{Compliance with Ethical Standards}

Conflict of Interest The authors declare that they have no conflict of interest.

Human and Animal Rights and Informed Consent This article does not contain any studies with human or animal subjects performed by any of the authors.

\section{References}

Papers of particular interest, published recently, have been highlighted as:

- Of importance

- Of major importance

1. Indrarespati A. and K Renaldi. Prevention and management of refeeding syndrome. The Indonesian Journal of Gastroenterology. Hepatol Digestive Endosc. 2016;17:200-3.

2. Friedli N, Stanga Z, Sobotka L, et al. Revisiting the refeeding syndrome: results of a systematic review. Nutrition. 2017;34:151-60. The first systematic review of RFS.

3. Crook M, Hally V, Panteli J. The importance of the refeeding syndrome. Nutrition. 2001;17(7-8):632-7. https://doi.org/10.1016/ S0899-9007(01)00542-1.

4. Mehanna H, Moledina J, Travis J. Refeeding syndrome: what it is, and how to prevent and treat it. BMJ. 2008;336(7659):1495-8. https://doi.org/10.1136/bmj.a301. This paper helps define RFS.

5. Marik P, Bedigian M. Refeeding hypophosphatemia in critically ill patients in an intensive care unit: a prospective study. Arch Surg. 1996;131:1043-7.

6. Camp M, Allon M. Severe hypophosphatemia in hospitalized patients. Mineral \& Electrolyte Metabolism. 1990;16:365-8.

7. Rio A, Whelan K, Goff L, et al. Occurrence of refeeding syndrome in adults started on artificial nutrition support: prospective cohort study. BMJ. 2013;3:eoo2173. https://doi.org/10.1001/archsurg. 1996.01430220037007.

8. Gonzalez A, Fajardo R, Gonzalez F. The incidence of the refeeding syndrome in cancer patients who receive artificial nutritional treatment. Nutr Hosp. 1996;11(2):98-101.

9. Chen J, Fan C. Prevention and management of refeeding syndrome in patients with chronic critical illness. 2016;19(7):737-9.

10. Serin, et al. Thiamin and folic acid deficiency accompanied by resistant electrolyte imbalance in the re-feeding syndrome in an elderly patient. Asia Pac J Clin Nutr. 2017;26(2):379-82.

11.• McClave S, et al. Guidelines for the provision and assessment of nutrition support therapy in the adult critically ill patient: Society of Critical Care Medicine (SCCM) and American Society for Parenteral and Enteral Nutrition (A.S.P.E.N.). JPEN. 2016;40(2): 159-211. https://doi.org/10.1177/0148607115621863 Guidelines to assist in clinical care and appropriate nutrition care.

12. Manon C, et al. Nutritional assessment of critically ill patients: validation of the modified NUTRIC score. Eur J Clinl Nutr. 2018;72:428-35. Paper showing the only validated score that can identify critically ill patients who would benefits from nutritional therapy.

13. Mcray S, Walker S, Parrish C. Much ado about refeeding. Practical Gastroenterology. 2004;28(12):26-44.

14. Aubry, et al. Refeeding syndrome in the frail elderly population: prevention, diagnosis and management. Clin Exp Gastroenterol. 2018;11:255-64.

15. Quiming C, Qiu L. Thiamine deficiency (Beriberi) induced polyneuropathy and cardiomyopathy: case report and review of the literature. J Med Cases. 2014;5(5):3018-311.

16. Reuler J, Girard D, Cooney T. Wernicke's encephalopathy. N Engl J Med. 1985;312(16):1035-9.

17. Zeki S, et al. Refeeding hypophosphataemia is more common in enteral than parenteral feeding in adult in patients. Clin Nutr. 2011 Jun;30(3):365-8.

18.• Doig, et al. Restricted versus continued standard caloric intake management of refeeding syndrome in critically ill adults: a randomized, parallel-group, multicenter, single-blind controlled trial. 
Lancet. 2015;3(12):943-52 Compares standard to hypocaloric feeding and published results.

19. Olthof L, Koekkoek W, van Setten C, et al. Impact of caloric intake in critically ill patients with and without, refeeding syndrome: a retrospective study. Clinical Nutrition. 2017. https://doi.org/10. 1016/j.clnu.2017.08.001.

20. Boot R, et al. Refeeding syndrome: relevance for the critically ill patient. Curr Opin Crit Care. 2018;24:235-40. https://doi.org/10. 1097/MCC.0000000000000514. Explains the results of permissive hypocaloric feeding.

21. Jo H, et al. The impact of multidisciplinary nutritional team involvement on nutritional care and outcomes in a medical intensive care unit. EJCN. 2017;71:1360-2.

22. Tappenden $\mathrm{K}$, et al. Critical role of nutrition in improving quality of care: an interdisciplinary call to action to address adult hospital malnutrition. Journal of the Academy of Nutrition and Dietetics. 2013;113(9):1219-37.

23.• National Institute for Health and Clinical Excellence. Nutrition support in adults Clinical guideline CG32. 2006; www.nice.org.uk/ page. $\operatorname{asp} x ? \mathrm{o}=\operatorname{cg} 032$. NICE criteria identify and stratify patients who are at risk of RFS.

24. Khan L, Ahmed J, MacFie J. Refeeding syndrome: a literature review. Gastroenterol Res Pract. 2010:2011. https://doi.org/10. 1155/2011/410971.

25. van Zanten A. Nutritional support and refreeding syndrome in critical illness. Lancet Respir Med. 2015;3:904-5.

Publisher's Note Springer Nature remains neutral with regard to jurisdictional claims in published maps and institutional affiliations. 